\title{
A Fatal Case of Body Packing: The Usefulness of the Postmortem CT before the Autopsy
}

\author{
Visonà SD ${ }^{1 *}$, Sozzi $\mathrm{M}^{2}$, Re $\mathrm{L}^{2}$, Castelli $\mathrm{F}^{2}$, Andrello \\ $\mathbf{L}^{3}$, Valeggia $\mathbf{P R}^{4}$ and Osculati $\mathbf{A}^{1}$ \\ ${ }^{1}$ Department of Public Health, Experimental and Forensic \\ Medicine, University of Pavia, Italy \\ ${ }^{2}$ Department of Bio-Technologies and Life Sciences, \\ University of Insubria, Italy \\ ${ }^{3}$ Service of Legal Medicine of Canton Ticino, Switzerland \\ ${ }^{4}$ Scientific Police of Canton Ticino, Switzerland \\ *Corresponding author: Silvia Damiana Visonà, \\ Department of Public Health, Experimental and Forensic \\ Medicine, University of Pavia. Address: via Forlanini 12, \\ 27100 Pavia, Italy
}

Received: June 05, 2017; Accepted: J une 23, 2017; Published: July 21, 2017

\begin{abstract}
The term body packing refers to the act of swallowing a high number of packages containing a great amount of illicit drugs, and concealing them in the lumen of the gastrointestinal tract in order to transport the illegal substance without being caught by security officers.

Even though drug abuse is generally a growing social issue, in Europe body packing is regarded as uncommon. Furthermore, this phenomenon often goes unrecognized, because of the difficulties in detecting the presence of drug packets inside the gastrointestinal tract.
\end{abstract}

Fatal cases have been seldom reported in literature. Yet, as the drug packets can accidentally leak or break inside the body, causing acute intoxication, forensic pathologists should consider this cause of death (often sudden and unexpected in a person previously healthy). As the authors point out by presenting this case, it is of great importance to perform preliminary radiological exams on the corpse, to serve as a guide during autopsy.

The subject of the present study is a young man coming from Africa, who was found dead in his hotel room. In the suspect of body packing, the corpse was submitted to a multi-slice total-body CT scan before the autopsy, showing the great interest and the usefulness of this approach.

Keywords: Body packer; Drug abuse; Drug trafficking; Heroin; Postmortem computed tomography

\section{Introduction}

"Body packers", "swallowers", "stuffers" or "mules" are some of the many terms that refer to people who illegally transport narcotic drugs concealing them in a body cavity [1]. Even though drug abuse is a growing social phenomenon, in northern Italy and in southern Switzerland (author's area of work), body packing is considered uncommon. Indeed, this phenomenon often goes unrecognized, because the presence of drug packets inside the body of an alive person can be difficult to detect. In fact, these packets are metal-free, so they cannot be detected by airport metal detectors. Moreover, sniffer dogs are unable to identify body packers.

Therefore, further knowledge about this phenomenon is necessary in order to find out how to promptly recognize a case of drug smuggling enacted in this way.

From a clinical point of view, it is essential to detect a body packer because drug packets can accidentally leak or break inside the body, causing sub-acute or acute intoxication, due to an absorption of the substance [2-9]. Fatal cases are unusual, but the forensic community should be aware of this cause of death. When there is the suspicion that the subject of forensic investigation was a drug courier, or the anamnesis is positive for signs of intoxication or symptoms of mechanical complications due to the packets, the forensic pathologist should consider the possibility of body packing, and perform preliminary radiological exams on the corpse which can be helpful as a guide during the autopsy [10-15].

\section{Materials and Methods}

The subject of the study was a 35-year-old man, who was found dead in his hotel room.

A forensic autopsy was performed 48 hours after death. Before the autopsy, anamnesis and some circumstantial data were acquired from the police records.

Then, a CT SIEMENS Somaton 16 Slices was used to perform a Computed Tomography (CT) Scan with a 3D reconstruction.

During the autopsy, samples for histological examinations were collected. The samples for the histology (brain, hypophysis, heart and pericardium, lungs, spleen, liver, kidney, adrenal glands) were formalin fixed for two days and then paraffin embedded. After having cut 5 micron slides, each specimen was stained with Hematoxylin Eosin.

Toxicology: Gas Chromatography/mass spectrometry was used to perform a biochemical analysis on the content of the packets. Gas Chromatography/mass Spectrometry (GC-MS), High Performance Liquid Chromatography with Diode Array Detector (HPLC-DAD), Headspace Gas Chromatography Flame Ionization Detector (HSGC-FID) was used to quantify drugs in blood and fluids.

\section{Case Presentation}

A young black man, who had arrived by plane from Tanzania the day before, was found dead in his hotel room. The afternoon before, 


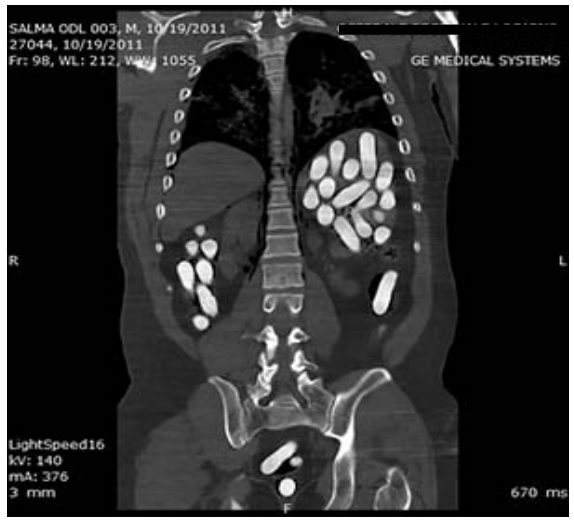

Figure 1: 2D CT-scan: frontal plane.

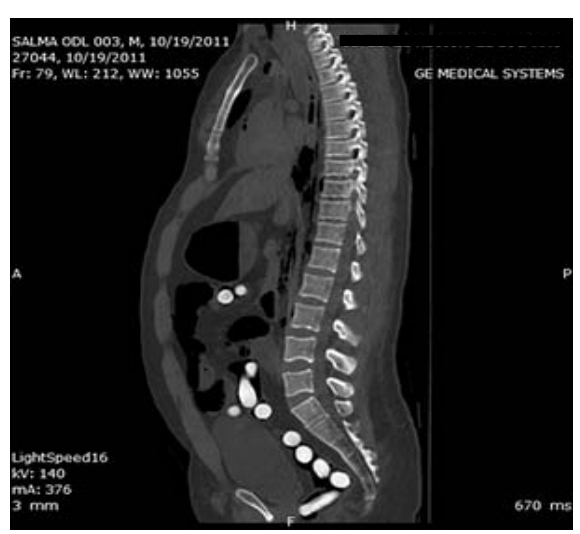

Figure 2: 2D CT-scan: sagittal plane.

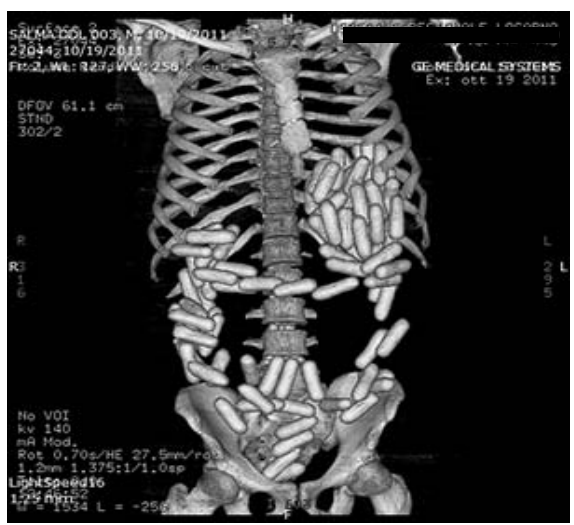

Figure 3: CT-scan 3D reconstruction: frontal view.

the man had been assisted at a train station, because he showed symptoms compatible with opiates abuse. He was transported to a local hospital, where the doctors documented: drowsiness, bilateral miosis and an episode of blood oxygen desaturation $\left(\mathrm{SaO}_{2} 75 \%\right)$. Physicians diagnosed opiates intoxication and treated the man with Naloxone. His general condition rapidly improved, so he refused further medical care and he left the hospital, against the doctors' advice. Some witnesses attested that immediately after having left the hospital, the African man had gone to the hotel, where he was found dead the morning after.

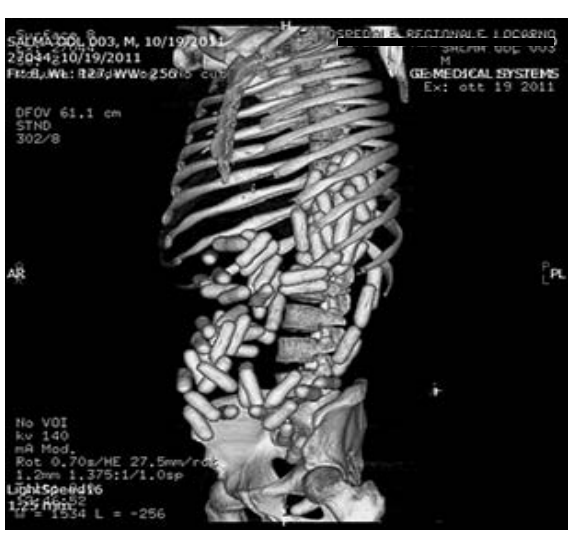

Figure 4: CT-scan 3D reconstruction: left side view.

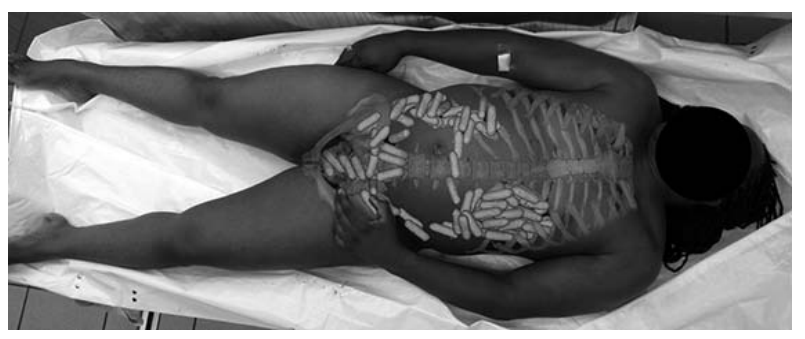

Figure 5: The corpse after the overlap of a frontal 3D image.

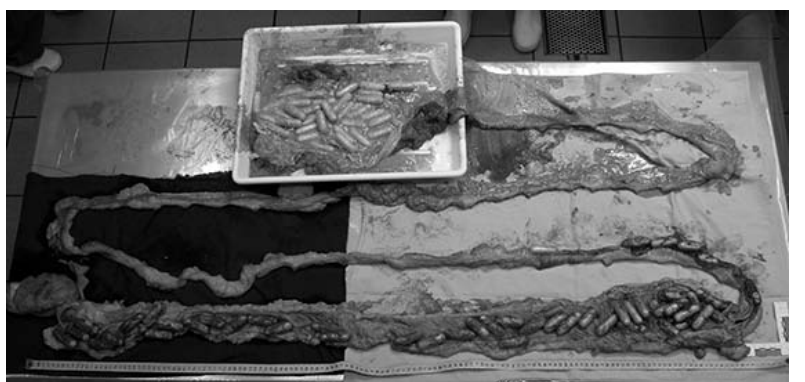

Figure 6: The whole gastro-intestinal tract opened.

The scene investigation revealed that the corpse lay on the bed on his back. Two opened milk cartons were found on the table, and an empty pizza box was found on the floor.

Knowing the geographical origin of the man, his recent clinical history and having suspicions of drug smuggling, a multi-slice totalbody CT scan exam was performed before the autopsy. This exam showed the presence of many egg-shaped objects distributed across the man's whole gastrointestinal tract, from his stomach down to his rectum, located, above all, in the stomach and in the large bowel (Figure 1-5). found.

The forensic autopsy was performed two days after the corpse was

The well-preserved body was $163 \mathrm{~cm}$ long and it weighted $96 \mathrm{~kg}$. The external examination of the corpse did not reveal any macroscopic evidence of trauma; both the mouth and the nose contained blood.

Before starting the autopsy, an overlap of a frontal 3D CT image with the body's picture has been made, in order to have a first view of 
Table 1: Biochemical analysis of six ovoid packets.

Table 1: Biochemical analysis of six ovoid packets.
\begin{tabular}{|c|c|c|c|l|}
\hline Sample & Narcotics & Purity [\%] & SD & Othersubstancesidentified \\
\hline Nr.1 & Heroin & 15.5 & 0.1 & Paracetamol, Caffeine, Glucose, Dextromethorphane, Lactose \\
\hline Nr.2.22 & Heroin & 14.7 & 0.4 & Paracetamol, Caffeine, Glucose, Dextromethorphane, Lactose \\
\hline Nr.2.42 & Heroin & 14.9 & 0.1 & Paracetamol, Caffeine, Glucose, Dextromethorphane, Lactose \\
\hline Nr.3.41 & Heroin & 14.4 & 0.2 & Paracetamol, Caffeine, Glucose, Dextromethorphane, Lactose \\
\hline Nr.4.1 & Heroin & 14.5 & 0.4 & Paracetamol, Caffeine, Glucose, Dextromethorphane, Lactose \\
\hline Nr.4.32 & Heroin & 15.1 & 0.3 & Paracetamol, Caffeine, Glucose, Dextromethorphane, Lactose \\
\hline
\end{tabular}

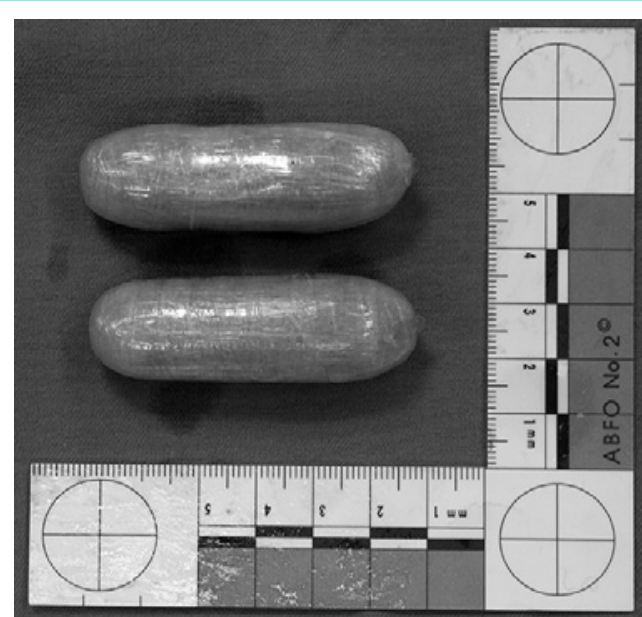

Figure 7: Two of the eighty-seven ovoid packets.

the ovoid objects' location into the gastrointestinal tract (Figure 5).

Upon opening the abdominal cavity, the stomach appeared distended and lumpy. The whole gastrointestinal tract was isolated and removed. The whole digestive tract, from the oesophagus to the rectum, was dissected, revealing the presence of overall 87 ovoid packets (Figure 6). In the stomach there were 34 packets, mixed with yellowish fluid with some whitish corpuscles. The first tract of small intestine was empty; the last $50 \mathrm{~cm}$ of small intestine contained 6 ovoid packets, mixed with faecal greenish fluid material. In the large intestine, 46 packets were found, mixed with small faecal greenish material. Another ovoid packet was found in the terminal tract of the rectum, near the anal sphincter. Each of these packets was $6 \mathrm{~cm}$ length, with a diameter of $1.5 \mathrm{~cm}$. They were covered by cellophanelike material, they had a hard consistency and all of them seemed to be intact (Figure 7). These packets were all extracted and handed over to the Police for further forensic analyses.

In the trachea, haematic and alimentary materials was found. Besides the severe congestion and oedema of the lungs, acute hematic congestion was also detected in the liver, adrenals and kidneys. The histological findings confirmed an intense congestion involving lungs, liver and kidneys.

After the autopsy, toxicological analyses were performed in order to find out the content of the eighty-seven ovoid packets. Each packet contained approximately $15 \mathrm{~g}$ of light brown, dump powder. In all, the 87 ovoid packets contained a total of $1316.03 \mathrm{~g}$ of powder. The subsequent analysis showed that the powder was heroin with a mean concentration of $14.8 \% \pm 0.49$ (Table 1). It was therefore found that the degree of purity of this substance was much lower compared with literature data. In fact, in drug smuggling, publications made by authors of different countries all over the world, show degrees of purity of heroin in the range of 50-90\% [15-17].

A drug screening conducted on samples of blood, collected during the autopsy, found lethal levels of morphine (in the urine there was a morphine total concentration of $45,000 \mu \mathrm{g} / \mathrm{l}$ and a codeine concentration of $3,700 \mu \mathrm{g} / \mathrm{l}$; in blood samples there was a morphine total concentration of $220 \mu \mathrm{g} / \mathrm{l}$ and a codeine concentration of 22 $\mu \mathrm{g} / \mathrm{l})$.

The postmortem investigations and the subsequent exams allowed identifying the cause of the man's death as acute heroin intoxication.

\section{Literature Review}

The trafficking of illicit drugs by swallowing packets and concealing them in the lumen of the gastrointestinal system was firstly reported in 1973, when a courier transported a condom filled with hashish from Lebanon to Canada [18]. This patient, with intestinal obstruction 13 days after ingesting hashish contained in a condom, recovered completely after a surgical operation. The first reported case of cocaine body packing was reported in 1975, in Florida [19]. At that time, cocaine had been identified as the illegal substance most frequently transported by body smuggling. Cocaine is still one of the most traded drugs, followed by heroin [20]. Many other kinds of substances, such as amphetamines, methylenedioxymethamphetamine ("ecstasy"), marijuana and hashish, are transported by ingestion and concealing within body cavities [1].

During the last 20 years, drug smuggling has increased globally and, in particular, the gastrointestinal tract has been used more frequently as a vehicle for smuggling drugs [20].

Typically, condoms, latex gloves, and balloons are used as packets for swallowing and subsequent retention in the body [20].

Usually, body packers carry 50 to 100 packets [1]. Each packet contains 8-12 g of substance of different purity, with smugglers on average carrying $1 \mathrm{~kg}$ (up to around $2 \mathrm{~kg}$ ) [21]. The packets are usually durable and tight and they can be both handmade and mechanically made; the typical size can vary between 2 and $8 \mathrm{~cm} \mathrm{[22].}$

The majority of body packers carry solid drugs, although body packers carrying liquid cocaine filled packages have increasingly been reported recently [22].

Median age is 30 years old ( 21 to 56 ) with a male/female ratio of 4.7 [23], while children, pregnant women or dogs have also been used $[1,24,25]$. 
Body packing can present many adverse events due to mechanical complications, such as gastrointestinal obstruction [26,27] or perforation [28], that can lead to death without surgical intervention [29].

The most serious toxic adverse effect, often fatal, may emerge as a result of leaky or ruptured packages. The acute fatal intoxication in a body packer was first described by Wetli and Mittleman in 1981 and it was called body packer syndrome [30]. This condition, also indicated with the term "toxidrome" [21], has been seldom reported in literature $[15,17,31]$.

In a forensic context, some points are worthy of highlighting. First, usually body packers, in an effort to transport illicit drugs to target destinations without being caught by security officers (even though they feel sick due to intoxication, both during the journey and once they arrive) conceal their symptoms, and may be found dead without a clinical history. Even when interrogated, most body packers may not act honestly, despite knowing the number and content of packages they carry. Therefore, they may be treated as sudden unexpected deaths and in such cases the pathologist starts the autopsy without any lead. On the contrary, this condition should be known by the forensic professional and considered, in order to conduct the postmortem examination properly.

The second remarkable point is the high lethality of this activity, as the contents of a single packet of drug usually exceed the acute oral lethal dose by many times, and therefore explains the great interest in this issue for the forensic community.

In the suspicion of body packing in an alive person, several imaging examinations can be enacted. The only fast and radiationfree procedure is ultrasonography; however, its sensitivity is low and a negative ultrasound examination cannot exclude the presence of ingested drug packets [32]. The simplest procedure is the abdominal radiography, which is also quick and cheap. Many studies evaluated the sensibility of X-Ray in a range of $40-90 \%$ [33], others in a range of $85-90 \%$ [34]. Both abdominal X-ray and ultrasonography can reportedly be used as screening tests [22].

The most accurate imaging method is CT Scan, which is now considered the most trustworthy method, with a sensibility of 95.6$100 \%[20,32,34]$. This technique is less frequently used because of the higher dose of radiation used than in X-Ray. However, the use of lowdose CT protocols permits to use of the same amount of radiation as in X-Ray and many studies demonstrated that these protocols are as useful as normal CT to detect the ingestion of drug packets [35-37]. CT scan is the preferred confirmatory imaging method, for example in highly suspected cases when abdominal plain film fails to make the diagnosis. The size and localization and number of packages can be precisely determined [22]. Moreover, rupture of package sheathes and drug leaks can also be demonstrated. There are several specific imaging signs characteristic of drug packets demonstrated in plain abdominal radiographs or CT scan. The packets are usually spherical or oval of a special conformity and rarely confused with scybala if arranged like a pearl chain [20]. Radiologists can find one or multiple well-defined opacities within the gastrointestinal tract. The appearance of drug packets homogeneously distributed in the bowel loops or stomach is called "tic-tac sign". Other commonly seen features are the "double condom sign", which is a definite crescent of air surrounding an ovoid opacity, and the "parallelism sign", when the packets are arranged in parallel within the bowel lumen $[34,37,38]$. Depending on the kind and the pureness of the narcotic, three particular patterns of attenuation have been illustrated: hashish is heavier than stool; cocaine appears comparable to stool; and heroin has an airy transparence [20].

The magnetic resonance imaging (MRI) has limited value in detecting drug packets because of the lack of protons and motion artefacts produced by the bowel loops [34]. The bowel must be immobilized with spasmolytic agents prior to MR study to reduce artifacts created by peristalsis, and it is not convenient in a forensic context. Yet, MRI can be of interest in the post mortem imaging, despite the high cost.

Even though radiological exams applied to alive people are well known, post mortem imaging has not be reported, to our knowledge, in cases of death due to body packing.

\section{Discussion and Conclusion}

In the present case, the acute fatal heroin intoxication was due to a leak of the substance contained in the packets, with absorption of heroin through bowel's mucosa, as confirmed by the toxicological analysis.

This case is similar to several other cases reported in literature, except concerning the medium concentration of heroin $(14.8 \% \pm$ 0.4 ), which was much lower if compared with literature data, which reports a degree of purity of heroin into the range of 50-90\% [15-17].

The most important message brought out by the present case is the great usefulness of the postmortem CT scan before the autopsy. The availability of CT images, in a case of drug smuggling, is essential to find out the presence and localization of the drug packets and to guide the autopsy, facilitating also the preservation and collection of the packets. Moreover, the precise site of the bowel where the rupture of packages occurred, which is extremely difficult to point out during the autopsy, can also be demonstrated.

Furthermore, in the present case, the $3 \mathrm{D}$ reconstruction was particularly helpful. This technique, followed by the autopsy, should be used in cases in which there is a suspicion of body packing. It may be particularly helpful when it comes to decomposed or diffusely damaged bodies (e.g. burnt bodies). An operative protocol should be applied in such cases, similar to what happens in a clinical context. Finally, the collaboration between the radiologist and the forensic pathologist is essential.

\section{References}

1. Traub SJ, Hoffman RS, Nelson LS. Body packing--the internal concealment of illicit drugs. N Engl J Med. 2003; 349: 2519-2526.

2. Silverberg D, Menes T, Kim U. Surgery for "body packers"--a 15-year experience. World J Surg. 2006; 30: 541-546.

3. Caruana DS, Weinbach B, Goerg D, Gardner LB. Cocaine-packet ingestion. Diagnosis, management, and natural history. Ann Intern Med. 1984; 100: 7374.

4. Glass JM, Scott HJ. 'Surgical mules': the smuggling of drugs in the gastrointestinal tract. J R Soc Med. 1995; 88: 450-453.

5. Swan MC, Byrom R, Nicolaou M, Paes T. Cocaine by internal mail: two surgical cases. J R Soc Med. 2003; 96: 188-189. 
6. Naseem A, Abbas S. Diacetylmorphine (heroin) body packer presenting with respiratory arrest. J Coll Physicians Surg Pak. 2009; 19: 262-263.

7. Shadnia S, Faiaz-Noori MR, Pajoumand A, Talaie H, Khoshkar A, VosoughGhanbari S, et al. A case report of opium body packer; review of the treatment protocols and mechanisms of poisoning. Toxicol Mech Methods. 2007; 17: 205-214.

8. Olumbe AK, Kalebi AY. Death from body packer syndrome: case report. East Afr Med J. 2004; 81: 218-220.

9. Stewart A, Heaton ND, Hogbin B. Body packing--a case report and review of the literature. Postgrad Med J. 1990; 66: 659-661.

10. Sengupta A, Page P. Window manipulation in diagnosis of body packing using computed tomography. Emerg Radiol. 2008; 15: 203-205.

11. Yang RM, Li L, Feng J, Lai SS, Lin BQ, Yu T, et al. Heroin body packing: clearly discerning drug packets using CT. South Med J. 2009; 102: 470-475.

12. Hahn IH, Hoffman RS, Nelson LS. Contrast CT scan fails to detect the last heroin packet. J Emerg Med. 2004; 27: 279-283.

13. Karkos PD, Cain AJ, White PS. An unusual foreign body in the oesophagus. The body packer syndrome. Eur Arch Otorhinolaryngol. 2005; 262: 154-156.

14. Hartoko TJ, Demey HE, De Schepper AM, Beaucourt LE, Bossaert LL. The body packer syndrome--cocaine smuggling in the gastro-intestinal tract. Klin Wochenschr. 1988; 66: 1116-1120.

15. Sribanditmongkol P, Supasingsiripreecha W, Thampitak S, Junkuy A. Fata heroin intoxication in body packers in northern Thailand during the last decade: two case reports. J Med Assoc Thai. 2006; 89: 106-110.

16. Mandava N, Chang RS, Wang JH, Bertocchi M, Yrad J, Allamaneni S, et al Establishment of a definitive protocol for the diagnosis and management of body packers (drug mules). Emerg Med J. 2011; 28: 98-101.

17. Wetli CV, Rao A, Rao VJ. Fatal heroin body packing. Am J Forensic Med Pathol. 1997; 18: 312-318.

18. Deitel M, Syed AK. Intestinal obstruction by an unusual foreign body. Can Med Assoc J. 1973; 109: 211-212

19. Mebane C, DeVito JJ. Cocain intoxication: a unique case. J Fla Med Assoc. 1975; 62: 19-20.

20. Reginelli A, Russo A, Urraro F, Maresca D, Martiniello C, D’Andrea A, et al. Imaging of body packing: errors and medico-legal issues. Abdom Imaging. 2015; 40: 2127-2142.

21. Berger FH, Nieboer KH, Goh GS, Pinto A, Scaglione M. Body packing: a review of general background, clinical and imaging aspects. Radiol Med 2015; 120: 118-132.

22. Bulakci $M$, Cengel $F$. The role of radiology in diagnosis and management of drug mules: an update with new challenges and new diagnostic tools. $\mathrm{Br} \mathrm{J}$ Radiol. 2016; 89: 20150888.

23. Veyrie N, Servajean S, Aissat A, Corigliano N, Angelakov C, Bouillot JL.
Value of a systematic operative protocol for cocaine body packers. World J Surg. 2008; 32: 1432-1437.

24. Goertemoeller S, Behrman A. The risky business of body packers and body stuffers. J Emerg Nurs. 2006; 32: 541-544.

25. Traub SJ, Kohn GL, Hoffman RS, Nelson LS. Pediatric "body packing”. Arch Pediatr Adolesc Med. 2003; 157: 174-177.

26. Freed TA, Sweet LN, Gauder PJ. Case reports balloon obturation bowel obstruction: a hazard of drug smuggling. AJR Am J Roentgenol. 1976; 127: 1033-1034.

27. Gómez Antúnez M, Cuenca Carvajal C, FarfánSedano A, Villalba MV, del Toro Cervera J, García Castaño J. Complications of intestinal transporting of cocaine packets. Study of 215 cases. Med Clin. 1998; 111: 336-337.

28. Joynt BP, Mikhael NZ. Sudden death of a heroin body packer. J Anal Toxicol. 1985; 9: 238-240.

29. Hutchins KD, Pierre-Louis PJ, Zaretski L, Williams AW, Lin RL, Natarajan GA. Heroin body packing: three fatal cases of intestinal perforation. J Forensic Sci. 2000; 45: 42-47.

30. Wetli CV, Mittlemann RE. The "body packer syndrome"-toxicity following ingestion of illicit drugs packaged for transportation. J Forensic Sci. 1981; 26: 492-500.

31. Gill JR, Graham SM. Ten years of "body packers" in New York City: 50 deaths. J Forensic Sci. 2002; 47: 843-846.

32. Pinto A, Reginelli A, Pinto F, Sica G, Scaglione M, Berger FH, et al. Radiological and practical aspects of body packing. Br J Radiol. 2014; 87: 20130500.

33. Cengel F, Bulakci M, Selcuk T, Savas Y, Ceyhan M, Kocak A, et al. The role of ultrasonography in the imaging of body packers comparison with $\mathrm{CT}$ : a prospective study. Abdom Imaging. 2015; 40: 2143-2151.

34. Ab Hamid S, Abd Rashid SN, Mohd Saini S. Characteristic imaging features of body packers: a pictorial essay. Jpn J Radiol. 2012; 30: 386-392.

35. Maurer MH, Niehues SM, Schnapauff D, Grieser C, Rothe JH, Waldmüller D, et al. Low-dose computed tomography to detect body-packing in an animal model. Eur J Radiol. 2011; 78: 302-306.

36. Beauverd Y, Poletti PA, Wolff H, Ris F, Dumonceau JM, Elger BS. A bodypacker with a cocaine bag stuck in the stomach. World J Radiol. 2011; 3: 155-158.

37. Poletti PA, Canel L, Becker CD, Wolff H, Elger B, Lock E, et al. Screening of illegal intracorporeal containers ("body packing"): is abdominal radiography sufficiently accurate? A comparative study with low-dose CT. Radiology. 2012; 265: 772-779.

38. Niewiarowski S, Gogbashian A, Afaq A, Kantor R, Win Z. Abdominal X-ray signs of intra-intestinal drug smuggling. J Forensic Leg Med. 2010; 17: 198202.
Austin J Forensic Sci Criminol - Volume 4 Issue 3 - 2017 ISSN : 2380-0801 | www.austinpublishing group.com Visonà et al. (C) All rights are reserved
Citation: Visonà SD, Sozzi M, Re L, Castelli F, Andrello L, Valeggia PR, et al. A Fatal Case of Body Packing: The Usefulness of the Postmortem CT before the Autopsy. Austin J Forensic Sci Criminol. 2017; 4(3): 1067. 